\title{
TIME-DEPENDENT EXACTLY SOLVABLE MODELS FOR QUANTUM COMPUTING
}

\author{
A. A. Suzko ${ }^{a, b, 1}$, G. Giorgadze ${ }^{a}$ \\ ${ }^{a}$ Joint Institute for Nuclear Research, Dubna \\ ${ }^{b}$ JIPENP, National Academy of Sciences of Belarus, Minsk
}

\begin{abstract}
A time-dependent periodic Hamiltonian admitting exact solutions is applied to construct a set of universal gates for quantum computer. The time evolution matrices are obtained in an explicit form and used to construct logic gates for computation. A way of obtaining entanglement operator is discussed, too. The method is based on transformation of soluble time-independent equations into time-dependent ones by employing a set of special time-dependent transformation operators.

Периодически зависящий от времени гамильтониан, допускающий точные решения, используется для построения универсального набора квантовых вентилей для квантовых компьютеров. Показано, как конструировать логические гейты на основе полученных в явном виде матриц эволюции. Обсуждается также способ получения операторов запутывания. Метод основан на преобразовании стационарной задачи в нестационарные с помощью специальных зависящих от времени операторов преобразования.
\end{abstract}

PACS: 02.70.-C; 03.67.Lx

\section{INTRODUCTION}

Recent studies of quantum computation have attracted considerable interest in both theoretical and experimental physics. The physical realization of the qubit register and a universal set of one-qubit and two-qubit logic gates is an important problem of quantum computation [1-3]. In this paper we shall construct one-qubit and two-qubit gates with desired properties controlled by time-dependent Hamiltonian.

A quantum computer is composed of a set of qubits which can be manipulated in a controlled way. Any quantum two-level systems can be taken to create qubits. A computation process corresponds to the evolution of the set of the qubits according to a specific unitary operator, for example, evolution operator $U(t)$. A general operation is decomposed into a discrete sequence in time of operations - quantum gates. The simplest unit of quantum information is a quantum bit, or qubit. The qubit is a vector in a two-dimensional Hilbert space, which can be presented as $|\psi\rangle=\alpha|0\rangle+\beta|1\rangle$. The basis vectors $|0\rangle$ and $|1\rangle$ are chosen as $|0\rangle=\left(\begin{array}{l}1 \\ 0\end{array}\right), \quad|1\rangle=\left(\begin{array}{l}0 \\ 1\end{array}\right), \quad|\psi\rangle=a\left(\begin{array}{l}1 \\ 0\end{array}\right)+b\left(\begin{array}{l}0 \\ 1\end{array}\right)=\left(\begin{array}{l}a \\ b\end{array}\right)$. Here $\alpha$ and $\beta$ are complex

\footnotetext{
${ }^{1}$ E-mail: suzko@1xpub01.jinr.ru
} 
coefficients, which satisfy the condition $|\alpha|^{2}+|\beta|^{2}=1$. Then $|\psi\rangle$ is the normalized vector, and $\alpha^{2}$ and $\beta^{2}$ characterize the probabilities of the results $|0\rangle$ and $|1\rangle$, correspondingly. The 2nd order matrices $\mathcal{U}(2 \times 2)$ transform one-qubit states and describe their evolution in time:

$$
\left|\psi_{f}\right\rangle=\mathcal{U}(2 \times 2)\left|\psi_{0}\right\rangle, \quad \mathcal{U}(t)=\left(\begin{array}{ll}
u_{11} & u_{12} \\
u_{21} & u_{22}
\end{array}\right) .
$$

Such transformations in quantum computation determine one-qubit quantum operations quantum gates.

The formalism of quantum mechanics is usually applied not to individual systems but to ensembles of systems. In quantum computation, the state of the computer of $n$ qubits can be expressed as a vector $|\Psi\rangle$ in a space of dimension $2^{n}$. Vector $|\Psi\rangle$ of the quantum register from $n$ qubits is expressed as a complex linear superposition of $2^{n}$ basis states:

$$
|\Psi\rangle=\sum_{k=0}^{2^{n}-1} a_{k}\left|j_{k}\right\rangle
$$

Here $a_{k}$ are projections of the vector $|\Psi\rangle$ on the directions of basis states $\left|j_{0}\right\rangle,\left|j_{1}\right\rangle, \ldots,\left|j_{2^{n}-1}\right\rangle$, $\sum_{k} a_{k}^{2}=1$. Basis states $|j\rangle=\left|i_{1}, i_{2}, \ldots, i_{n}\right\rangle=\left|i_{1}\right\rangle \otimes\left|i_{2}\right\rangle \otimes \ldots\left|i_{n}\right\rangle, \quad i_{1}, i_{2}, \ldots, i_{n}=\{0,1\}$ are presented as

$$
\begin{aligned}
& \left|j_{0}\right\rangle=|0\rangle \otimes|0\rangle \ldots \otimes|0\rangle \\
& \left|j_{1}\right\rangle=|0\rangle \otimes|0\rangle \ldots \otimes|1\rangle \\
& \left.\left|j_{\left.2^{n}-1\right\rangle}=\right| 1\right\rangle \otimes|1\rangle \ldots \otimes|1\rangle
\end{aligned}
$$

The transformation of an initial state vector $\left|\Psi_{0}\right\rangle$ into the final vector $\left|\Psi_{f}\right\rangle$ models the process of calculation on quantum computer

$$
\left|\Psi_{f}\right\rangle=\mathcal{U}\left(2^{n} \times 2^{n}\right)\left|\Psi_{0}\right\rangle
$$

Vectors $\left|\Psi_{0}\right\rangle$ and $\left|\Psi_{f}\right\rangle$ are vectors in the $2^{n}$ Hilbert space. The transformation matrices $\mathcal{U}\left(2^{n} \times 2^{n} ; t\right)$ define the dynamic evolution of the quantum system from $n$ qubits. At the same time, the matrices $\mathcal{U}\left(2^{n} \times 2^{n} ; t\right)$ provide the process of quantum computing at each fixed moment.

Clearly, the realization of the transformation $\mathcal{U}\left(2^{n} \times 2^{n}\right)$ with $n>3$ is a very difficult problem. As usual, one considers the presentation of $\mathcal{U}\left(2^{n} \times 2^{n}\right)$ as a production of second $U(2 \times 2)$ order and forth $U(4 \times 4)$ order matrices:

$$
\mathcal{U}\left(2^{n} \times 2^{n}\right)=\prod_{i, j} \mathcal{U}_{i}(2 \times 2) \otimes \mathcal{U}_{j}\left(2^{2} \times 2^{2}\right) .
$$

As is known [1], a universal set of gates is given by $2 \times 2$ unitary operators and a unitary entangled operator $4 \times 4$ which acts on $C^{2} \otimes C^{2}$. We shall show how it is possible to generate explicitly one-qubit logic gates from the time evolution matrices and give a way of obtaining entanglement operators. 


\section{A UNIVERSAL GATE SET}

The universal one-qubit logic gates can be constructed from the time evolution matrices which we obtain in a closed analytic form. In our approach, the time-dependent periodic Hamiltonians admitting exact solutions are applied to control the time evolution of the onequbit gates. The time-dependent Hamiltonians are obtained from time-independent soluble Hamiltonians and a set of unitary time-dependent transformations [4].

Suppose that the time evolution of the quantum system is governed by the Schrödinger equation

$$
i \frac{\partial|\Psi(r, t)\rangle}{\partial t}=H(r, t)|\Psi(r, t)\rangle
$$

with $\hbar=1$ and $T$ periodic time-dependent Hamiltonian, $H(t)=H(t+T)$.

Assume that the initial state of the qubit can be written in one of the states of the time-independent Hamiltonian $\widetilde{H}$ :

$$
\widetilde{H}=\boldsymbol{\sigma} \cdot \widetilde{\mathbf{B}}=\lambda\left(\begin{array}{cc}
\cos \widetilde{\theta} & \sin \widetilde{\theta} \\
\sin \widetilde{\theta} & -\cos \widetilde{\theta}
\end{array}\right),
$$

$\phi_{1}=\cos \tilde{\theta} / 2|0\rangle+\sin \theta / 2|1\rangle$ or $\phi_{2}=-\sin \theta / 2|0\rangle+\cos \tilde{\theta} / 2|1\rangle$. Taking the gauge transformation as

$$
|\Psi(r, t)\rangle=\mathcal{S}(t)|\Phi(r, t)\rangle, \quad S(t)=\exp \left(-i \sigma_{x} \omega_{1} t / 2\right),
$$

the time-independent Hamiltonian (5) is changed to the time-dependent one:

$$
H(t)=\mathcal{S}(t) \widetilde{H} \mathcal{S}^{\dagger}(t)+i \dot{\mathcal{S}}(t) \mathcal{S}^{\dagger}(t)
$$

The evolution operator $U(t)=\exp \left(-i \sigma_{x} \omega_{1} t / 2\right) \exp (-i \widetilde{H} t)$, corresponding to the timedependent Hamiltonian

$$
H(t)=\lambda\left(\begin{array}{cc}
\cos \tilde{\theta} \cos \left(\omega_{1} t\right) & \sin \tilde{\theta}-\omega_{1} / 2 \lambda+i \cos \tilde{\theta} \sin \left(\omega_{1} t\right) \\
\sin \tilde{\theta}-\omega_{1} / 2 \lambda-i \cos \tilde{\theta} \sin \left(\omega_{1} t\right) & -\cos \widetilde{\theta} \cos \left(\omega_{1} t\right)
\end{array}\right),
$$

is written as

$$
U_{1}(t)=\left(\begin{array}{cc}
\cos \left(\omega_{1} t / 2\right) & -i \sin \left(\omega_{1} t / 2\right) \\
-i \sin \left(\omega_{1} t / 2\right) & \cos \left(\omega_{1} t / 2\right)
\end{array}\right)\left(\begin{array}{cc}
\exp (-i \lambda t) & 0 \\
0 & \exp (i \lambda t)
\end{array}\right) .
$$

The time evolution matrix $U(t)$ is the universal one-qubit gate, which is controlled by the time-dependent magnetic field parameters $\omega_{1}$ and $\lambda$.

An important one-bit transformation is the operation of negation or inversion operation NOT $=\sigma_{x}$. The gate NOT can be obtained from (8) at $\omega_{1} t=\pi$ and $\lambda t=2 n \pi$ and then after multiplication of the result by $i$ :

$$
\mathrm{NOT}=i U_{1}\left(\omega_{1} t=\pi, \lambda t=2 n \pi\right)=\left(\begin{array}{ll}
0 & 1 \\
1 & 0
\end{array}\right) .
$$


The transformation NOT exchanges $|0\rangle$ and $|1\rangle$, e.g., NOT $(a|0\rangle+b|1\rangle)=a|1\rangle+b|0\rangle$. Another special one-qubit gate can be obtained from (8) at $\omega_{1} t=\pi$ and $\lambda t=\pi / 2$ and after multiplication of the result by $i$ :

$$
\mathrm{Y}=i U_{1}\left(\omega_{1} t=\pi, \lambda t=\pi / 2\right)=\left(\begin{array}{cc}
0 & -i \\
i & 0
\end{array}\right)=\sigma_{y}
$$

The special gate $Z$ is obtained from (8) at $\omega_{1} t=4 \pi$ and $\lambda t=\pi / 2$ and after multiplication by $i$ :

$$
\mathrm{Y}=i U_{1}\left(\omega_{1} t=4 \pi, \lambda t=\pi / 2\right)=\left(\begin{array}{cc}
1 & 0 \\
0 & -1
\end{array}\right)=\sigma_{z} .
$$

Now let us obtain another important single-bit transformation. It is the Hadamard transformation defined by

$$
\mathrm{H}=\frac{1}{\sqrt{2}}\left(\begin{array}{cc}
1 & 1 \\
1 & -1
\end{array}\right)=\frac{1}{\sqrt{2}}\left(\sigma_{x}+\sigma_{z}\right) .
$$

When applied to $|0\rangle$ and to $|1\rangle, \mathrm{H}$ creates the superposition of states with the equal probability

$$
\mathrm{H}|0\rangle=H\left(\begin{array}{l}
1 \\
0
\end{array}\right)=\frac{1}{\sqrt{2}}(|0\rangle+|1\rangle), \quad H|1\rangle=H\left(\begin{array}{l}
0 \\
1
\end{array}\right)=\frac{1}{\sqrt{2}}(|0\rangle-|1\rangle) .
$$

If the initial state of the qubit is $|0\rangle$, then the evolution matrix $U(t)$ corresponding to the time-dependent Hamiltonian (8) is written as

$$
\begin{aligned}
U(t)=\exp \left(-i \sigma_{x} \omega_{1} t / 2\right) \exp \left(-i \sigma_{z} \lambda t\right) \exp \left(-i \sigma_{y} \widetilde{\theta} / 2\right)= \\
=\left(\begin{array}{cc}
\cos \left(\omega_{1} t / 2\right) & -i \sin \left(\omega_{1} t / 2\right) \\
-i \sin \left(\omega_{1} t / 2\right) & \cos \left(\omega_{1} t / 2\right)
\end{array}\right)\left(\begin{array}{cc}
\exp (-i \lambda t) & 0 \\
0 & \exp (i \lambda t)
\end{array}\right) \times \\
\times\left(\begin{array}{cc}
\cos (\widetilde{\theta} / 2) & -\sin (\tilde{\theta} / 2) \\
\sin (\tilde{\theta} / 2) & \cos (\tilde{\theta} / 2)
\end{array}\right) .
\end{aligned}
$$

At $t=0, \widetilde{\theta}=\pi / 2$ and any $\omega_{1}, \lambda$, from (13) we obtain the gate

$$
U\left(\omega_{1}, \lambda ; t=0, \widetilde{\theta}=\pi / 2\right)=\frac{1}{\sqrt{2}}\left(\begin{array}{cc}
1 & -1 \\
1 & 1
\end{array}\right) .
$$

To obtain the Hadamard gate, we multiply NOT by the gate $U\left(\omega_{1}, \lambda ; t=0, \widetilde{\theta}=\pi / 2\right)$. Therefore, the Hadamard gate $\mathrm{H}$ is a result of the sequence of two transformations:

$$
\mathrm{H}=i U_{1}(\pi, 2 \pi n, \widetilde{\theta}=0) U\left(\omega_{1}, \lambda, \widetilde{\theta}=\pi / 2 ; t=0\right) .
$$

Here $U_{1}(t)=U(t ; \widetilde{\theta}=0)$ was used. Applied to $n$ bits, $\mathrm{H}$ generates superposition of all $2^{n}$ possible states, which can be considered as a binary representation of the numbers from 0 to $2^{n}-1$ :

$$
\begin{aligned}
& (H \otimes H \otimes \ldots \otimes H)|00 \ldots 0\rangle= \\
& =\frac{1}{\sqrt{2^{n}}}((|0\rangle+|1\rangle) \otimes(|0\rangle+|1\rangle) \otimes \ldots \otimes(|0\rangle+|1\rangle))=\frac{1}{\sqrt{2^{n}}} \sum_{k=0}^{2^{n}-1}\left|j_{k}\right\rangle .
\end{aligned}
$$


1.1. Construction of Two-Qubit Gates. The 2 nd order matrices $\mathcal{U}_{i}(2 \times 2)$ transform one-qubit states. The 4 th order matrices $\mathcal{U}_{j}\left(2^{2} \times 2^{2}\right)$ transform couples of one-qubit states. There are four basis states in 4th dimension Hilbert space for two-qubit systems building on one-qubit states $|0\rangle,|1\rangle$ :

$$
\begin{aligned}
& \{|00\rangle=|0\rangle \otimes|0\rangle,|01\rangle=|0\rangle \otimes|1\rangle,|10\rangle=|1\rangle \otimes|0\rangle,|11\rangle=|1\rangle \otimes|1\rangle\}, \\
& |00\rangle=\left(\begin{array}{l}
1 \\
0 \\
0 \\
0
\end{array}\right),|01\rangle=\left(\begin{array}{l}
0 \\
1 \\
0 \\
0
\end{array}\right),|10\rangle=\left(\begin{array}{l}
0 \\
0 \\
1 \\
0
\end{array}\right),|11\rangle=\left(\begin{array}{l}
0 \\
0 \\
0 \\
1
\end{array}\right) .
\end{aligned}
$$

Any two-qubit state can be expressed as a superposition of these basis states:

$$
|\Psi\rangle=c_{00}|00\rangle+c_{10}|10\rangle+c_{01}|01\rangle+c_{11}|11\rangle,
$$

where $\left|c_{00}\right|^{2}+\left|c_{01}\right|^{2}+\left|c_{10}\right|^{2}+\left|c_{11}\right|^{2}=1$.

Entanglement. A gate $G$ is said to be entangling, if $|\Psi\rangle=G\left|\psi_{1}\right\rangle \otimes\left|\psi_{2}\right\rangle$ is not decomposable as a tensor product of two one-qubit states. If in (17) $c_{00} c_{11}-c_{01} c_{10} \neq 0$, then $|\Psi\rangle$ is an entangled state. The property $\left|\Psi_{12}\right\rangle \neq\left|\psi_{1}\right\rangle \otimes\left|\psi_{2}\right\rangle$ is called entanglement. In our case the entanglement operator is obtained from two independent systems with the use of unitary gauge time-dependent transformations, which lead to time-dependent periodic operators and entanglement of states.

One of the important two-qubit gates is the Controlled NOT=CNOT gate, which can be defined by

$$
\mathrm{CNOT}=|0\rangle\langle 0|\otimes \mathbf{1}+| 1\rangle\langle 1| \otimes \sigma_{x}=\left(\begin{array}{cccc}
1 & 0 & 0 & 0 \\
0 & 1 & 0 & 0 \\
0 & 0 & 0 & 1 \\
0 & 0 & 1 & 0
\end{array}\right) .
$$

1.2. Construction of the Hamiltonian with the Desired Entangled Operator. Let

$$
H=h \otimes 1+1 \otimes h+\epsilon A,
$$

where $\epsilon \in\{0,1\}$ and $h$ is a two-dimensional diagonal time-independent Hamiltonian in the form $h=\left(\begin{array}{ll}a & 0 \\ 0 & b\end{array}\right)$. The evolution operator of the matrix Schrödinger equation (4) with the Hamiltonian (19) is expressed as follows:

$$
U(t)=\left(\mathrm{e}^{-i h t} \otimes \mathrm{e}^{-i h t}\right) \mathrm{e}^{-i A t},
$$

if the operator $A$ commutes with the Hamiltonian $h \otimes 1+1 \otimes h$. We would like to get the entanglement operator $U(t)$ and to construct a corresponding Hamiltonian in the form (19). To this end, let us select the operator $R(t)=\mathrm{e}^{-i A t}$ in the form

$$
R(t)=\left(\begin{array}{cccc}
1 & 0 & 0 & 0 \\
0 & \cos (t) & -i \sin (t) & 0 \\
0 & -i \sin (t) & \cos (t) & 0 \\
0 & 0 & 0 & 1
\end{array}\right)
$$


Find $A(t)$ from

$$
A=i \frac{d R(t)}{d t} R^{-1}(t)=\left(\begin{array}{cccc}
0 & 0 & 0 & 0 \\
0 & 0 & 1 & 0 \\
0 & 1 & 0 & 0 \\
0 & 0 & 0 & 0
\end{array}\right)
$$

The matrix $h=\sigma_{3} / 2=\frac{1}{2}\left(\begin{array}{cc}1 & 0 \\ 0 & -1\end{array}\right)$ satisfies the condition of commutation $[A,(h \otimes 1+1$ $\otimes h)]$. At last, substitution of $\mathrm{e}^{-i A t}$ and $h$ into the evolution matrix $U(t)$ gives the entanglement operator

$$
U(t)=\left(\begin{array}{cccc}
\mathrm{e}^{i t} & 0 & 0 & 0 \\
0 & \cos (t) & -i \sin (t) & 0 \\
0 & -i \sin (t) & \cos (t) & 0 \\
0 & 0 & 0 & \mathrm{e}^{-i t}
\end{array}\right)
$$

So, the entanglement operator has been obtained with the use of the unitary time-dependent transformation (20), which leads to the time-dependent periodic operator $U(t)$ and entanglement of states. We obtain the corresponding Hamiltonian (19) with $A$ as given in (21).

Acknowledgements. This work has been partially supported by a grant of the Russian Foundation for Basic Research 06-01-00228.

\section{REFERENCES}

1. Bryglinski J.L., Bryglinski R. Universal Quantum Gates in Mathematics of Quantum Computation. Boca Ratton, Florida: Chapman and Hall/ CRC Press, 2002.

2. Kauffman L. H. Braiding Operators are Universal Quantum Gates. quant-ph/0401090. 2004.

3. Radtke T., Fritzshe S. // Comp. Phys. Commun. 2005. V.173. P. 91.

4. Suzko A. A. // Phys. Lett. A. 2003. V.308. P. 267. 